
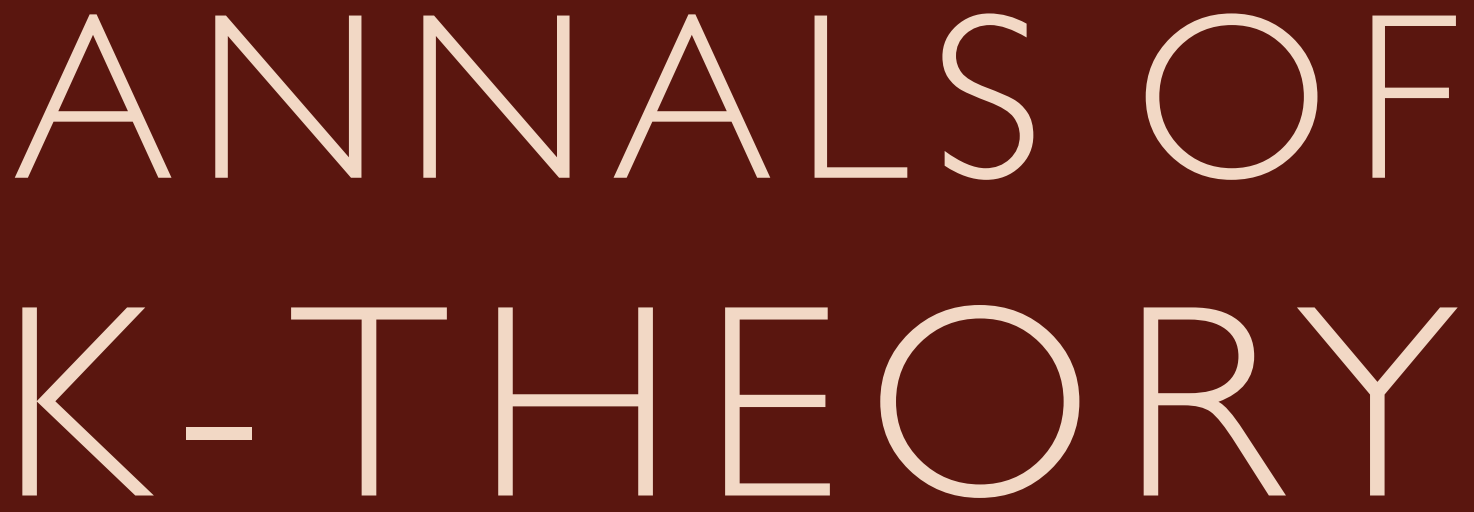

$$
\text { vol. } 5 \text { no. } 32020
$$

The Topological Period-Index Conjecture for $\operatorname{spin}^{c}$ 6-manifolds

Diarmuid Crowley and Mark Grant

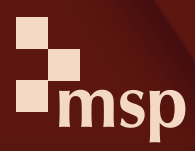

A JOURNAL OF THE K-THEORY FOUNDATION 



\title{
The Topological Period-Index Conjecture for $\operatorname{spin}^{c} 6$-manifolds
}

\author{
Diarmuid Crowley and Mark Grant
}

\begin{abstract}
The Topological Period-Index Conjecture is a hypothesis which relates the period and index of elements of the cohomological Brauer group of a space. It was identified by Antieau and Williams as a topological analogue of the Period-Index Conjecture for function fields.

In this paper we show that the Topological Period-Index Conjecture holds and is in general sharp for $\operatorname{spin}^{c} 6$-manifolds. We also show that it fails in general for 6-manifolds.
\end{abstract}

\section{Introduction}

This paper is about the Topological Period-Index Problem (TPIP), which was identified by Antieau and Williams [2014a; 2014b] as an important analogue of periodindex problems in algebraic geometry. We give a brief introduction to the TPIP and refer the reader to [Antieau and Williams 2014a; 2014b] for more information.

Let $X$ be a connected space with the homotopy type of a finite $C W$-complex. The cohomological Brauer group of $X$ is defined to be the torsion subgroup of its third integral cohomology group:

$$
\operatorname{Br}^{\prime}(X):=T H^{3}(X) .
$$

Here and throughout integer coefficients are omitted. For $\alpha \in \operatorname{Br}^{\prime}(X)$, the period of $\alpha$ is defined to be the order of $\alpha$,

$$
\operatorname{per}(\alpha):=\operatorname{ord}(\alpha) .
$$

Let $P U(n):=U(n) / U(1)$ be the $n$-dimensional projective unitary group, which is the quotient of the unitary group $U(n)$ by its centre. By a theorem of Serre [Grothendieck 1968, Corollaire 1.7], every class $\alpha \in T H^{3}(X)$ arises as the obstruction to lifting the structure group of some principal $P U(n)$-bundle $P \rightarrow X$ to the group $U(n)$. In this case one writes $\alpha=\delta(P)$ and defines the index of $\alpha$ by

$$
\operatorname{ind}(\alpha):=\operatorname{gcd}(n: \alpha=\delta(P) \text { for a } P U(n) \text {-bundle } P),
$$

MSC2010: primary 57R19; secondary 14F22, 19L50.

Keywords: Brauer groups, twisted $K$-theory, period-index problems. 
so that the index defines the homotopy-invariant function

$$
\text { ind }: T H^{3}(X) \rightarrow \mathbb{Z}, \quad \alpha \mapsto \operatorname{ind}(\alpha) \text {. }
$$

From the definitions, one sees that $\operatorname{per}(\alpha) \mid$ ind $(\alpha)$ and by [Antieau and Williams 2014a, Theorem 3.1] the primes dividing $\operatorname{per}(\alpha)$ and ind $(\alpha)$ coincide. The TPIP is the problem of relating the index of a class $\alpha$ to its period and properties of $X$, like its dimension.

To investigate the TPIP, Antieau and Williams [2014b, Straw Man] formulated what is often called the Topological Period-Index Conjecture (TPIC) for X:

TPIC. If $X$ is homotopy equivalent to a $C W$-complex of dimension $2 d$ and if $\alpha$ is an element of $\operatorname{Br}^{\prime}(X)$, then

$$
\operatorname{ind}(\alpha) \mid \operatorname{per}(\alpha)^{d-1}
$$

Warning. The TPIC should be regarded as a hypothesis for investigating the TPIP and not as a conjecture, in the usual sense of the word.

Indeed, while the obstruction theory developed by Antieau and Williams [2014b, Theorem A] shows that the TPIC holds for any 4-dimensional complex, they also prove that the TPIC fails in general for 6-dimensional complexes, but at most by a factor of two.

Theorem 1.1 (cf. [Antieau and Williams 2014b, Theorems A and B]). Let $X$ be $a$ 6-dimensional $C W$-complex, $\alpha \in \mathrm{Br}^{\prime}(X)$ have period $n$, and $\operatorname{set} \epsilon(n):=\operatorname{gcd}(n, 2)$. Then $\operatorname{ind}(\alpha) \mid \epsilon(n) n^{2}$.

Moreover, if $X$ is a 6-skeleton of the Eilenberg-Mac Lane space $K(\mathbb{Z} / 2,2)$ and we take the generator $\alpha \in H^{3}(X)=\mathbb{Z} / 2$ (so that $\left.\operatorname{per}(\alpha)=2\right)$, then $\operatorname{ind}(\alpha)=8>$ $\operatorname{per}(\alpha)^{2}$.

An important motivation for Antieau and Williams in identifying the TPIC was the Algebraic Period-Index Conjecture (APIC) which was identified in the work of Colliot-Thélène [2002]. This is a statement in algebraic geometry concerning the Brauer group of certain algebras $A$. When $A=\mathbb{C}(V)$ is the function field of a smooth complex variety $V$, then the APIC for $\mathbb{C}(V)$ implies the TPIC for $V$. When the variety $V$ has complex dimension $d=1$, the APIC is trivially true, it was proven for $d=2$ by de Jong [2004], and for $d \geq 3$ we have the Antieau-Williams alternative:

(A) either there exits a $V$ violating the TPIC, in which case the APIC fails in general,

(B) or every $V$ satisfies the TPIC (in which case we have identified an a priori new topological property of smooth complex varieties). 
In this paper we show that for $d=3$ the latter statement holds. This may be regarded as evidence for the APIC in complex dimension 3.

A smooth complex projective variety $V$ is in particular a manifold: here and besides Remark 1.9, we use the word "manifold" to mean "closed smooth manifold". Recall that a manifold $M$ admits a $\operatorname{spin}^{c}$ structure if it is orientable and the second Stiefel-Whitney class of $M$ has an integral lift. For example, every variety $V$ as above admits a $\operatorname{spin}^{c}$ structure. More generally, it is well known that a 6-manifold admits a $\operatorname{spin}^{c}$ structure if and only if it admits an almost complex structure (as can be easily deduced from results in [Massey 1961]).

Theorem 1.2. The Topological Period-Index Conjecture holds for $\operatorname{spin}^{c}$ 6-manifolds.

As we explain in Section 2, Theorem 1.2 is an elementary consequence of results of Antieau and Williams [2014b] and:

Theorem 1.3. Let $N$ be a closed $\operatorname{spin}^{c} 6$-manifold and let $x \in H^{2}(N ; \mathbb{Z} / 2)$. Then there exists a class $e_{x} \in H^{2}(N)$ such that

$$
\beta^{\mathbb{Z} / 2}\left(x^{2}\right)=\beta^{\mathbb{Z} / 2}(x) e_{x} \in H^{5}(N),
$$

where $\beta^{\mathbb{Z} / 2}: H^{*}(N ; \mathbb{Z} / 2) \rightarrow H^{*+1}(N)$ denotes the mod 2 Bockstein.

To discuss the TPIP further for 6-manifolds we recall that Teichner [1995] has already constructed orientable 6-manifolds $N$ with $x \in H^{2}(N ; \mathbb{Z} / 2)$ such that $\beta^{\mathbb{Z} / 2}\left(x^{2}\right) \neq 0$. The manifolds in Teichner's examples are all the total-spaces of 2 -sphere bundles over 4-manifolds, where the class $x$ restricts to a generator of $H^{2}\left(S^{2} ; \mathbb{Z} / 2\right)$. We call pairs $(N, x)$ coming from Teichner's examples Teichner pairs (see Definition 5.3) and investigating their construction we prove:

Theorem 1.4. For a Teichner pair $(N, x)$, let $\alpha:=\beta^{\mathbb{Z} / 2}(x) \in T H^{3}(N)$.

(1) If the base 4-manifold of a Teichner pair $(N, x)$ is orientable, then $N$ is $\operatorname{spin}^{c}$, $\operatorname{per}(\alpha)=2$, and ind $(\alpha)=4$.

(2) There exist Teichner pairs $(N, x)$ over nonorientable 4-manifolds where we have $\operatorname{per}(\alpha)=2$ but ind $(\alpha)=8$.

Summarising Theorems 1.2 and 1.4 we obtain the following result on the TPIP for 6-manifolds.

Theorem 1.5. The TPIC fails in general for 6-manifolds but it holds and is in general sharp for $\operatorname{spin}^{c}$ 6-manifolds.

Remark 1.6. One may view Theorem 1.3 as giving a cohomological obstruction to a closed 6-manifold admitting a $\operatorname{spin}^{c}$ structure. For instance, we do not currently know how to prove that the Teichner manifold $N$ appearing in Theorem 1.4(2) (and Proposition 5.9) is not $\operatorname{spin}^{c}$, except by invoking Theorem 1.3. 
Remark 1.7. The nonvanishing of $\beta^{\mathbb{Z} / 2}\left(x^{2}\right) \in H^{5}(N)$ is related to various nonrealisability phenomena, for which the examples in [Teichner 1995] are of minimal dimension. For example, $\beta^{\mathbb{Z} / 2}\left(x^{2}\right)$ vanishes if $x \in H^{2}(N ; \mathbb{Z} / 2)$ can be realised as the second Stiefel-Whitney class $w_{2}(E)$ of some real vector bundle $E$ over $N$, since $w_{2}(E)^{2}$ is the mod 2 reduction of the integral class $p_{1}(E)$, the first Pontrjagin class.

It is a classical result of Thom [1954] that $\beta^{\mathbb{Z} / 2}\left(x^{2}\right)$ vanishes if the Poincaré dual of $x$ in $H_{4}(N ; \mathbb{Z} / 2)$ is realised as the fundamental class of an embedded 4manifold in $N$. More recently, in [Grant and Szúcs 2013] the second author and Szúcs showed that $\beta^{\mathbb{Z} / 2}\left(x^{2}\right)$ vanishes if the Poincare dual of $x$ is realised by the fundamental class of an immersion of a 4-manifold in $N$ and more precisely that the Poincare dual of $\beta^{\mathbb{Z} / 2}\left(x^{2}\right)$ is realised by the singular set of a generic smooth map realising the Poincaré dual of $x$. Notwithstanding Remarks 1.6 and 1.8, the geometric significance of the condition $\beta^{\mathbb{Z} / 2}\left(x^{2}\right) \notin \beta^{\mathbb{Z} / 2}(x) H^{2}(N)$ appearing in Section 2 remains somewhat mysterious.

Remark 1.8. The TPIP also arises in twisted $K$-theory, where classes $\alpha \in T H^{3}(X)$ define the twisting used to define the $K$-groups, $K_{\alpha}^{*}(X)$, of $\alpha$-twisted vector bundles over $X$ [Donovan and Karoubi 1970]. For $\alpha \in T H^{3}(X)$ and $i: * \rightarrow X$ the inclusion of a point, by [Antieau and Williams 2014a, Proposition 2.21], we have

$$
i^{*}\left(K_{\alpha}^{0}(X)\right)=\operatorname{ind}(\alpha) K^{0}(*)=\operatorname{ind}(\alpha) \mathbb{Z} .
$$

Hence $\operatorname{ind}(\alpha)$ is the index of the intersection $\bigcap_{i=1}^{\infty} \operatorname{Ker}\left(d_{i}\right) \subseteq H^{0}\left(X ; K^{0}\right) \cong \mathbb{Z}$, where $d_{i}: H^{0}\left(X ; K^{0}\right) \rightarrow H^{i}\left(X ; K^{i-1}\right)$ is the $i$-th differential in the twisted AtiyahHirzebruch spectra sequence computing $K_{\alpha}^{*}(X)$.

This perspective is behind the index formula [Antieau and Williams 2014b, Theorem A], which we use in Section 2, and also the recent work of Gu [2019] on the TPIP for 8-complexes. Gu shows that the 3-primary TPIP for 8-complexes involves controlling $\beta^{\mathbb{Z} / 3}\left(x^{3}\right) / \beta^{\mathbb{Z} / 3}(x) H^{4}(X)$ for classes $x \in H^{2}(X ; \mathbb{Z} / 3)$, just as the TPIP for 6-complexes involves controlling $\beta^{\mathbb{Z} / 2}\left(x^{2}\right) / \beta^{\mathbb{Z} / 2}(x) H^{2}(X)$ for classes $x \in H^{2}(X ; \mathbb{Z} / 2)$. We expect that the methods of this paper involving the integrality of $\mathrm{Wu}$ classes and the bilinear algebra of the subsection beginning on page 613 will generalise to combine with the work of $\mathrm{Gu}$ and prove the TPIC for odd-order Brauer classes over orientable 8-manifolds.

Remark 1.9. It is natural to wonder whether the singular spaces $Z$ underlying singular complex 3-dimensional projective varieties satisfy the TPIC. In this direction, we note that the complement of the singular set in $Z$ can often be compactified to give a $\operatorname{spin}^{c}$ manifold with boundary $(N, \partial N)$. The arguments of this paper can be generalised to prove that if $(N, \partial N)$ is a compact $\operatorname{spin}^{c}$ manifold with boundary where the first Chern class of $N$ vanishes on $\partial N$ and $T H_{1}(\partial N) \otimes \mathbb{Z} / 2=0$, then 
the TPIC holds for quotients $N / \partial N$. As a consequence we believe that the TPIC holds for singular spaces underlying certain complex 3-dimensional varieties with isolated conical singularities.

Organisation. The rest of this paper is organised as follows. In Section 2 we prove Theorem 1.2 assuming Theorem 1.3. In Section 3 we establish some preliminary results about linking pairings and bilinear forms. In Section 4 we prove Theorem 1.3 and in Section 5 we discuss Teichner's examples and prove Theorem 1.4.

\section{The Topological Period-Index Conjecture for $\operatorname{spin}^{c}$ 6-manifolds}

In this section we prove that the Topological Period-Index Conjecture holds for $\operatorname{spin}^{c} 6$-manifolds. This is an elementary consequence of Theorem 1.3 and results in [Antieau and Williams 2014b].

Let $\alpha \in \operatorname{Br}^{\prime}(X)=T H^{3}(X)$ with ord $(\alpha)=n$ and let

$$
\beta^{\mathbb{Z} / n}: H^{*}(X ; \mathbb{Z} / n) \rightarrow H^{*+1}(X)
$$

be the $\bmod n$ Bockstein, which lies in the exact sequence

$$
H^{*}(X ; \mathbb{Z} / n) \stackrel{\beta^{\mathbb{Z} / n}}{\longrightarrow} H^{*+1}(X) \stackrel{\times n}{\longrightarrow} H^{*+1}(X) .
$$

As $\operatorname{ord}(\alpha)=n$, we see that $\alpha=\beta^{\mathbb{Z} / n}(\xi)$ for some $\xi \in H^{2}(X ; \mathbb{Z} / n)$. We consider the Pontrjagin square

$$
P_{2}: H^{2}(X ; \mathbb{Z} / 2 m) \rightarrow H^{4}(X ; \mathbb{Z} / 4 m)
$$

and following Antieau and Williams define $\widetilde{Q}(\xi) \in H^{5}(X) / \alpha H^{2}(X)$ by the equation

$$
\widetilde{Q}(\xi):= \begin{cases}{\left[\beta^{\mathbb{Z} / n}\left(\xi^{2}\right)\right]} & n \text { is odd, } \\ {\left[\beta^{\mathbb{Z} / 2 n}\left(P_{2}(\xi)\right)\right]} & n \text { is even, }\end{cases}
$$

where $[\gamma] \in H^{5}(X) / \alpha H^{2}(X)$ denotes the coset of $\gamma \in H^{5}(X)$. By [Antieau and Williams 2014b, Theorem A], the element $\widetilde{Q}(\xi)$ depends only on $\alpha$ and when $X$ is a 6-dimensional CW-complex,

$$
\operatorname{ind}(\alpha)=\operatorname{ord}(\widetilde{Q}(\xi)) \operatorname{per}(\alpha)
$$

Hence to verify the Topological Period-Index Conjecture in dimension 6, it suffices to show that $\operatorname{ord}(\widetilde{Q}(\xi)) \mid n$, i.e., $n \widetilde{Q}(\xi)=0$. For this we consider the commutative diagram

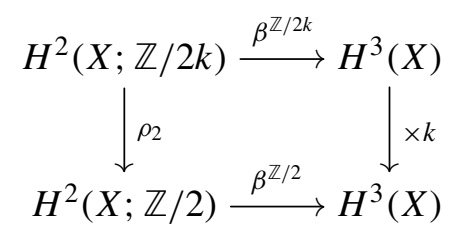


where $\rho_{2}$ denotes reduction modulo 2 and the diagram commutes as a consequence of the commutative diagram of coefficient short exact sequences

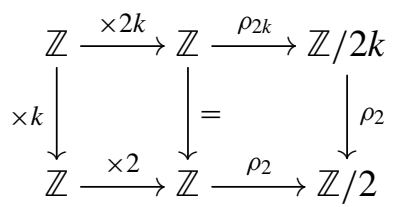

Hence for all $\xi \in H^{2}(X ; \mathbb{Z} / 2 k)$ we have the equation

$$
\beta^{\mathbb{Z} / 2}\left(\rho_{2}(\xi)\right)=k \beta^{\mathbb{Z} / 2 k}(\xi) .
$$

Proof of the Topological Period-Index Conjecture for $\operatorname{spin}^{c}$ 6-manifolds. Let $\left(N, c_{1}\right)$ be a $\operatorname{spin}^{c} 6$-manifold and $\alpha \in \operatorname{Br}^{\prime}(N)$ have order $n$, and choose $\xi \in H^{2}(N ; \mathbb{Z} / n)$ such that $\alpha=\beta^{\mathbb{Z} / n}(\xi)$. If $n$ is odd, then $n \widetilde{Q}(\xi)=0$ and so by [Antieau and Williams 2014b, Theorem A(3)] the Topological Period-Index Conjecture holds for $\alpha$. If $n=2 m$, then set

$$
x:=\rho_{2}(\xi) \in H^{2}(N ; \mathbb{Z} / 2) .
$$

By Theorem 1.3, there is a $y \in H^{2}(N)$ such that $\beta^{\mathbb{Z} / 2}\left(x^{2}\right)=\beta^{\mathbb{Z} / 2}(x) y$. Applying (2.1) we obtain

$$
\beta^{\mathbb{Z} / 2}\left(x^{2}\right)=\beta^{\mathbb{Z} / 2}(x) y=m \beta^{\mathbb{Z} / 2 m}(\xi) y=m \alpha y \in \alpha H^{2}(N) \subseteq H^{5}(N)
$$

and so $\left[\beta^{\mathbb{Z} / 2}\left(x^{2}\right)\right]=0 \in H^{5}(N) / \alpha H^{2}(N)$. Applying (2.1) and (2.2) we obtain

$$
\begin{aligned}
2 m \widetilde{Q}(\xi)=2 m\left[\beta^{\mathbb{Z} / 4 m}\left(P_{2}(\xi)\right)\right]=\left[2 m \beta^{\mathbb{Z} / 4 m}\left(P_{2}(\xi)\right)\right] \\
=\left[\beta^{\mathbb{Z} / 2}\left(\rho_{2}\left(P_{2}(\xi)\right)\right)\right]=\left[\beta^{\mathbb{Z} / 2}\left(x^{2}\right)\right]=0,
\end{aligned}
$$

where the second to last equality holds since $P_{2}$ satisfies $\rho_{2 m}\left(P_{2}(\xi)\right)=\xi^{2}$, where $\rho_{2 m}$ denotes reduction to modulo $2 m$.

\section{Linking pairings and bilinear forms}

In this section we establish some elementary results used in the proof of Theorem 1.3.

Some properties of Bockstein homomorphisms. For a space $X$ and a positive integer $n$ recall that

$$
\beta^{\mathbb{Z} / n}: H^{*}(X ; \mathbb{Z} / n) \rightarrow H^{*+1}(X)
$$

is the Bockstein associated to the coefficient sequence $\mathbb{Z} \rightarrow \mathbb{Z} \stackrel{\rho_{n}}{\rightarrow} \mathbb{Z} / n$.

Lemma 3.1. Let $x \in H^{*}(X ; \mathbb{Z} / n)$ and $y \in H^{i}(X)$, and consider $x y \in H^{*+i}(X ; \mathbb{Z} / n)$.

Then

$$
\beta^{\mathbb{Z} / n}(x y)=\beta^{\mathbb{Z} / n}(x) y .
$$


Proof. Let $y \in C^{i}(X)$ be a cocycle representative for $y$ and $\rho_{n}$ denote reduction modulo $n$, and consider the commutative diagram below, in which the rows are short exact sequences of chain complexes:

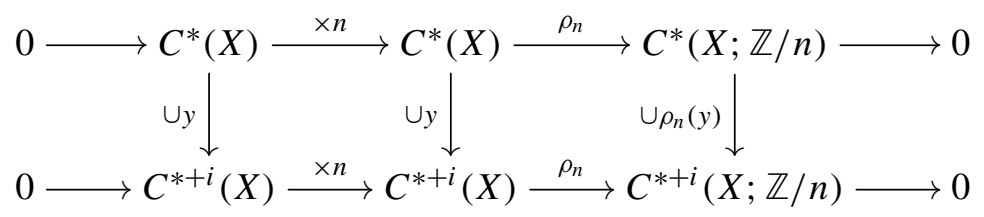

Observe that the vertical arrows are chain maps, since the coboundary is a derivation and $y$ is a cocycle. The result now follows from the naturality of connecting homomorphisms. (Compare to [Brown 1982, Chapter V, §3.3].)

We also consider the Bockstein homomorphism

$$
\beta^{\mathbb{Q} / \mathbb{Z}}: H^{*}(X ; \mathbb{Q} / \mathbb{Z}) \rightarrow H^{*+1}(X),
$$

which is associated to the coefficient sequence $\mathbb{Z} \rightarrow \mathbb{Q} \stackrel{\pi}{\rightarrow} \mathbb{Q} / \mathbb{Z}$. Let

$$
\iota_{n}: \mathbb{Z} / n \rightarrow \mathbb{Q} / \mathbb{Z}
$$

be the inclusion defined by sending $[1] \in \mathbb{Z} / n$ to $\left[\frac{1}{n}\right]$ and also write

$$
\iota_{n}: H^{*}(X ; \mathbb{Z} / n) \rightarrow H^{*}(X ; \mathbb{Q} / \mathbb{Z})
$$

for the map on homology induced by $\iota_{n}$. The commutative diagram of coefficient sequences

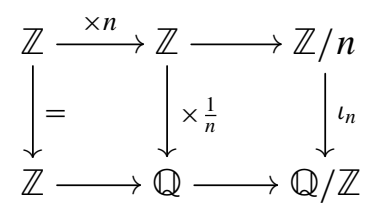

gives rise to the equality

$$
\beta^{\mathbb{Z} / n}=\beta^{\mathbb{Q} / \mathbb{Z}} \circ \iota_{n}: H^{*}(X ; \mathbb{Z} / n) \rightarrow H^{*+1}(X) .
$$

The linking pairings of an oriented manifold. Let $G$ and $H$ be finite abelian groups. Recall that a bilinear pairing

$$
\phi: G \times H \rightarrow \mathbb{Q} / \mathbb{Z}
$$

is called perfect if $g=0 \in G$ if and only if $\phi(g, h)=0$ for all $h \in H$ and $h=0 \in H$ if and only if $\phi(g, h)=0$ for all $g \in G$.

Remark 3.3. A useful property of perfect pairings, which we leave for the reader to verify, is that $h_{1}=h_{2} \in H$ if and only if $\phi\left(g, h_{1}\right)=\phi\left(g, h_{2}\right)$ for all $g \in G$. An analogous statement holds for $g_{1}, g_{2} \in G$. 
Now let $M$ be a closed, connected, oriented $m$-manifold with $[M] \in H_{m}(M)$ the fundamental class of $M$. For each $k=2, \ldots, m-2$, the linking pairing of $M$ is the pairing

$$
b_{M}: T H^{k+1}(M) \times T H^{m-k}(M) \rightarrow \mathbb{Q} / \mathbb{Z}, \quad(f, y) \mapsto b_{M}(f, y):=\langle\tilde{f} y,[M]\rangle,
$$

where $\tilde{f} \in H^{k}(M ; \mathbb{Q} / \mathbb{Z})$ is any class such that $\beta^{\mathbb{Q} / \mathbb{Z}}(\tilde{f})=f$. The pairing $b_{M}$ is well defined because if $\tilde{f}^{\prime}$ is some other lift of $f$, then $\tilde{f}-\tilde{f}^{\prime}$ itself lifts to $H^{k}(M ; \mathbb{Q})$ and then $\langle\tilde{f} y,[M]\rangle-\left\langle\tilde{f}^{\prime} y,[M]\right\rangle=\left\langle\left(\tilde{f}-\tilde{f}^{\prime}\right) y,[M]\right\rangle=0$, since $y$ is torsion.

Lemma 3.4. The linking pairing $b_{M}: T H^{k+1}(M) \times T H^{m-k}(M) \rightarrow \mathbb{Q} / \mathbb{Z}$ is a perfect pairing such that for all $w \in H^{k}(M ; \mathbb{Z} / n)$ and all $y \in T H^{m-k}(M)$

$$
b_{M}\left(\beta^{\mathbb{Z} / n}(w), y\right)=\iota_{n}(\langle w y,[M]\rangle) .
$$

Proof. That $b_{M}$ is perfect is well known. The case $m=2 k+1$ is part of [Davis and Kirk 2001, Exercise 55]. The general case follows from results in [Seifert and Threlfall 1934]. Since we did not find a definitive reference in the literature, we give a proof below.

For a finite abelian group $G$, let $G^{\wedge}:=\operatorname{Hom}(G, \mathbb{Q} / \mathbb{Z})$ denote the torsion dual of $G$. A pairing $\phi: G \times H \rightarrow \mathbb{Q} / \mathbb{Z}$ of finite abelian groups induces adjoint homomorphisms $\hat{\phi}: H \rightarrow G^{\wedge}, h \mapsto[g \mapsto \phi(g, h)]$ and $\hat{\phi}^{\prime}: G \rightarrow H^{\wedge}, g \mapsto[h \mapsto \phi(g, h)]$, and it is easily checked that $\phi$ is perfect if and only if either one of $\hat{\phi}$ or $\hat{\phi}^{\prime}$ is an isomorphism.

Standard properties of cup and cap products give $\langle\tilde{f} y,[M]\rangle=\langle\tilde{f}, y \cap[M]\rangle$. Hence the adjoint homomorphism of $b_{M}$,

$$
\hat{b}_{M}: T H^{m-k}(M) \rightarrow T H^{k+1}(M)^{\wedge}, \quad y \mapsto\left[f \mapsto b_{M}(f, y)=\langle\tilde{f}, y \cap[M]\rangle\right],
$$

is equal to the composition $\hat{\phi}_{M} \circ P D$, where $P D: T H^{m-k}(M) \rightarrow T H_{k}(M)$ is the Poincaré duality isomorphism and $\hat{\phi}_{M}: T H_{k}(M) \rightarrow T H^{k+1}(M)^{\wedge}$ is an adjoint of the pairing

$$
\phi_{M}: T H^{k+1}(M) \times T H_{k}(M) \rightarrow \mathbb{Q} / \mathbb{Z}, \quad(f, b) \mapsto\langle\tilde{f}, b\rangle,
$$

for $\tilde{f} \in H^{k}(M ; \mathbb{Q} / \mathbb{Z})$ a lift of $f$. Hence it suffices to prove that $\hat{\phi}_{M}$ is an isomorphism or equivalently that the other adjoint $\hat{\phi}_{M}^{\prime}: T H^{k+1}(M) \rightarrow T H_{k}(M)^{\wedge}$ is an isomorphism. Since the finite groups $T H^{k+1}(M)$ and $T H_{k}(M)^{\wedge}$ have the same order by the universal coefficient theorem, it suffices to show that $\hat{\phi}_{M}^{\prime}$ is injective.

Suppose that $\hat{\phi}_{M}^{\prime}(f)=0$ and let $\tilde{f} \in H^{k}(M ; \mathbb{Q} / \mathbb{Z})$ be a lift of $f$. Then for all $b \in T H_{k}(M)$

$$
\langle\tilde{f}, b\rangle=0 \in \mathbb{Q} / \mathbb{Z}
$$


Since $\mathbb{Q} / \mathbb{Z}$ is an injective $\mathbb{Z}$-module, another application of the universal coefficient theorem gives

$$
H^{k}(M ; \mathbb{Q} / \mathbb{Z}) \cong \operatorname{Hom}\left(H_{k}(M), \mathbb{Q} / \mathbb{Z}\right) \cong T H_{k}(M)^{\wedge} \oplus \operatorname{Hom}\left(F H_{k}(M) ; \mathbb{Q} / \mathbb{Z}\right),
$$

where $F H_{k}(M):=H_{k}(M) / T H_{k}(M)$. With respect to the above decomposition we have $\tilde{f}=(0, \bar{z})$ for some $\bar{z} \in \operatorname{Hom}\left(F H_{k}(M) ; \mathbb{Q} / \mathbb{Z}\right)$. Now $\bar{z}$ can be lifted to $z \in H^{k}(M ; \mathbb{Q})$ so that $\tilde{f}-\pi(z)=0$ but then $f=\beta^{\mathbb{Q} / \mathbb{Z}}(\tilde{f})=\beta^{\mathbb{Q} / \mathbb{Z}}(\tilde{f}-\pi(z))=0$ and so $\hat{\phi}_{M}^{\prime}$ is injective.

The second statement follows directly from the definition of $b_{M}$ and (3.2).

Bilinear forms over $\mathbb{Z} / 2$. In this subsection we establish a basic fact about symmetric bilinear forms over $\mathbb{Z} / 2$. Let $V$ be a finitely generated $(\mathbb{Z} / 2)$-vector space and let

$$
\lambda: V \times V \rightarrow \mathbb{Z} / 2
$$

be a symmetric bilinear form on $V$. If $V^{*}:=\operatorname{Hom}(V, \mathbb{Z} / 2)$ is the dual vector space to $V$, then the adjoint homomorphism of $\lambda$ is the homomorphism

$$
\hat{\lambda}: V \rightarrow V^{*}, \quad v \mapsto(w \mapsto \lambda(v, w)) .
$$

The form $(\lambda, V)$ is nonsingular if $\hat{\lambda}: V \rightarrow V^{*}$ an isomorphism. Notice that the map

$$
\gamma(\lambda): V \rightarrow \mathbb{Z} / 2, \quad v \mapsto \lambda(v, v)
$$

is linear since

$$
\begin{aligned}
\lambda(v+w, v+w)=\lambda(v, v) & +\lambda(v, w)+\lambda(w, v)+\lambda(w, w) \\
& =\lambda(v, v)+2 \lambda(v, w)+\lambda(w, w)=\lambda(v, v)+\lambda(w, w) .
\end{aligned}
$$

Thus $\gamma(\lambda) \in V^{*}$.

Lemma 3.5. For all $\lambda, \gamma(\lambda) \in \operatorname{Im}(\hat{\lambda})$.

Proof. For the orthogonal sum of bilinear forms, $\lambda_{0} \oplus \lambda_{1}$, we have

$$
\gamma\left(\lambda_{0} \oplus \lambda_{1}\right)=\gamma\left(\lambda_{0}\right) \oplus \gamma\left(\lambda_{1}\right)
$$

The lemma follows since every symmetric bilinear form over a finite field is isomorphic to the orthogonal sum of the zero form and a nonsingular form.

Remark 3.6. Although we will not use this fact, it is worthwhile to note that Lemma 3.5 is equivalent to the following statement: let $A$ be a symmetric matrix over $\mathbb{Z} / 2$; then the diagonal of $A$ lies in the column space of $A$. 
Example 3.7. Let $N$ be a closed, connected, oriented 6-manifold, and let $x \in$ $H^{2}(N ; \mathbb{Z} / 2)$. We identify $H^{6}(N ; \mathbb{Z} / 2)=\mathbb{Z} / 2$ and for the $(\mathbb{Z} / 2)$-vector space

$$
V:=T H^{2}(N) / 2 T H^{2}(N)
$$

we define the symmetric bilinear form

$$
\lambda_{x}: V \times V \rightarrow \mathbb{Z} / 2, \quad([y],[z]) \mapsto y x z .
$$

By Lemma 3.5, there is a vector $[d] \in V$ such that $\hat{\lambda}_{x}([d])=\gamma\left(\lambda_{x}\right) \in V^{*}$. Hence for any $d_{x} \in[d] \subset T H^{2}(N)$ and all $y \in T H^{2}(N)$, we have

$$
y^{2} x=y x y=\lambda_{x}([y],[y])=\lambda_{x}\left([y],\left[d_{x}\right]\right)=y x d_{x} .
$$

\section{The proof of Theorem $\mathbf{1 . 3}$}

Let $N$ be a closed, connected, oriented $\operatorname{spin}^{c}$ 6-manifold. To prove Theorem 1.3 it suffices to prove the following: for $x \in H^{2}(N ; \mathbb{Z} / 2)$ and all $y \in T H^{2}(N)$, there is a class $e_{x} \in H^{2}(N)$ such that

$$
x^{2} y=x e_{x} y \in H^{6}(N ; \mathbb{Z} / 2) .
$$

To see this we use the linking pairing of $N$, which is a perfect pairing by Lemma 3.4:

$$
b_{N}: T H^{5}(N) \times T H^{2}(N) \rightarrow \mathbb{Q} / \mathbb{Z} .
$$

From (4.1) and Lemmas 3.4 and 3.1, for all $y \in T H^{2}(N)$ we have

$$
\begin{aligned}
b_{N}\left(\beta^{\mathbb{Z} / 2}\left(x^{2}\right), y\right)=\iota_{2}\left(\left\langle x^{2} y,[N]\right\rangle\right)=\iota_{2}\left(\left\langle x e_{x} y,[N]\right\rangle\right) \\
\quad=b_{N}\left(\beta^{\mathbb{Z} / 2}\left(x e_{x}\right), y\right)=b_{N}\left(\beta^{\mathbb{Z} / 2}(x) e_{x}, y\right) .
\end{aligned}
$$

Thus $\beta^{\mathbb{Z} / 2}\left(x^{2}\right)=\beta^{\mathbb{Z} / 2}(x) e_{x}$, since $b_{N}$ is perfect; see Remark 3.3.

To find $e_{x}$, we start with $v_{2}(N)$, the second Wu class of $N$. Since $N$ is orientable, $v_{2}(N)$ coincides with $w_{2}(N)$, the second Stiefel-Whitney class of $N$. Since $N$ is $\operatorname{spin}^{c}$, the class $w_{2}(N)$ lifts to an integral class $c_{1} \in H^{2}(N)$. In summary, we have

$$
v_{2}(N)=w_{2}(N)=\rho_{2}\left(c_{1}\right) \in H^{2}(N ; \mathbb{Z} / 2) .
$$

By definition of the Wu class $v_{2}(N)$ we have

$$
x y v_{2}(N)=S q^{2}(x y)=x^{2} y+x y^{2},
$$

where we have used the Cartan formula for $S q^{2}(x y)$ and the fact that $S q^{1}\left(\rho_{2}(y)\right)=0$. By (4.2) we can replace $v_{2}(N)$ by $c_{1}$ in (4.3) and rearranging we obtain

$$
x^{2} y=x y c_{1}+x y^{2} .
$$


By Example 3.7, there is an element $d_{x} \in T H^{2}(N)$ such that $x y^{2}=x y d_{x}$ and so

$$
x^{2} y=x y c_{1}+x y d_{x}=x y e_{x}
$$

where $e_{x}:=c_{1}+d_{x}$. Hence we have found $e_{x}$ as in (4.1), finishing the proof of Theorem 1.3.

\section{Teichner's examples}

In this section we recall a construction due to Teichner [1995] which produces closed smooth 6-manifolds $N$ with classes $x \in H^{2}(N ; \mathbb{Z} / 2)$ such that $\beta^{\mathbb{Z} / 2}\left(x^{2}\right) \neq 0$. The manifolds $N$ are constructed as total spaces of sphere bundles of rank-3 vector bundles $E$ over closed 4-manifolds. In the following, $\mathbb{Z}^{w_{1}(E)}$ denotes integral coefficients twisted by the first Stiefel-Whitney class of the bundle $E$.

Lemma 5.1 [Teichner 1995, Lemma 1]. Let E be a 3-dimensional vector bundle over a path-connected space $X$, with sphere bundle $N=S E$.

(i) There exists a class $x \in H^{2}(N ; \mathbb{Z} / 2)$ which restricts to the generator in the cohomology $H^{2}\left(S^{2} ; \mathbb{Z} / 2\right)$ of the fibre if and only if $w_{3}(E)=0$.

(ii) Assume that $w_{2}(E)$ is not the reduction of a class in $H^{2}\left(X ; \mathbb{Z}^{w_{1}(E)}\right)$. Then any class $x$ as in (i) has $0 \neq \beta^{\mathbb{Z} / 2}\left(x^{2}\right) \in H^{5}(N ; \mathbb{Z})$.

The next lemma guarantees the existence of such bundles with base $X=M$ a closed connected 4-manifold.

Lemma 5.2 [Teichner 1995, Lemma 2]. Let $M$ be a closed connected 4-manifold with fundamental group $\mathbb{Z} / 4$. Then there exists a 3-dimensional bundle $E$ over $M$ with $w_{3}(E)=0, w_{1}(E)=w_{1}(M)$, and $w_{2}(E)$ not the reduction of a class in $H^{2}\left(M ; \mathbb{Z}^{w_{1}(E)}\right)$.

Definition 5.3. The total space $N$ of the sphere bundle of a bundle $E$ satisfying the conditions of Lemma 5.2 is a closed connected 6-manifold, which by Lemma 5.1 supports a class $x \in H^{2}(N ; \mathbb{Z} / 2)$ satisfying $\beta^{\mathbb{Z} / 2}\left(x^{2}\right) \neq 0$. We will call such a total space $N$ a Teichner manifold and the pair $(N, x)$ a Teichner pair.

Spin $^{c}$ 6-manifolds $N$ with $\beta^{\mathbb{Z} / 2}\left(x^{2}\right) \neq 0 \in H^{5}(N)$. In this subsection we show that a Teichner manifold over an orientable base is $\operatorname{spin}^{c}$.

Lemma 5.4. Let $N$ be a Teichner manifold over a closed connected 4-manifold $M$. Then

(i) $N$ is orientable,

(ii) and if $M$ is orientable, then $N$ is $\operatorname{spin}^{c}$. 
Proof. Let $\pi: N \rightarrow M$ be the bundle projection. Since the normal bundle of the sphere bundle in the total space of $E$ is trivial, there are bundle isomorphisms

$$
\left.T N \oplus \mathbb{R} \cong T E\right|_{N} \cong \pi^{*}(T M) \oplus \pi^{*}(E) .
$$

Now part (i) follows from the equation

$$
w_{1}(N)=\pi^{*} w_{1}(M)+\pi^{*} w_{1}(E)=0 .
$$

For (ii), assume $w_{1}(M)=0$ so that

$$
w_{2}(N)=\pi^{*} w_{2}(M)+\pi^{*} w_{1}(M) \pi^{*} w_{1}(E)+\pi^{*} w_{2}(E)=\pi^{*} w_{2}(M)+\pi^{*} w_{2}(E) .
$$

Then

$$
\beta^{\mathbb{Z} / 2}\left(w_{2}(N)\right)=\pi^{*}\left(\beta^{\mathbb{Z} / 2}\left(w_{2}(M)\right)\right)+\pi^{*}\left(\beta^{\mathbb{Z} / 2}\left(w_{2}(E)\right)\right) .
$$

The first term vanishes since any orientable 4-manifold is $\operatorname{spin}^{c}$; see [Morgan 1996] for example. The second term vanishes since $\beta^{\mathbb{Z} / 2}\left(w_{2}(E)\right) \in H^{3}(M)$ is the Euler class of the orientable bundle $E$.

The following proposition proves Theorem 1.4(i).

Proposition 5.5. Let $(N, x)$ be a Teichner pair over a closed, connected, orientable 4-manifold. Then $N$ is $\operatorname{spin}^{c}$ and $\beta^{\mathbb{Z} / 2}\left(x^{2}\right) \neq 0$, but $\beta^{\mathbb{Z} / 2}\left(x^{2}\right) \in \beta^{\mathbb{Z} / 2}(x) H^{2}(N)$.

Furthermore, the element $\alpha=\beta^{\mathbb{Z} / 2}(x) \in T H^{3}(N)$ has $\operatorname{per}(\alpha)=2$ and $\operatorname{ind}(\alpha)=4$.

Proof. The first statement is a consequence of Lemmas 5.4 and 5.1 and Theorem 1.3.

To prove the second statement, we recall that by [Antieau and Williams 2014b, Theorem A],

$$
\operatorname{ind}(\alpha)=\operatorname{ord}(\widetilde{Q}(x)) \operatorname{per}(\alpha)
$$

where $\widetilde{Q}(x)=\left[\beta^{\mathbb{Z} / 4}\left(P_{2}(x)\right)\right] \in H^{5}(N) / \alpha H^{2}(N)$. Note that by Theorem 1.3 and (2.1),

$$
2 \widetilde{Q}(x)=2\left[\beta^{\mathbb{Z} / 4}\left(P_{2}(x)\right)\right]=\left[2 \beta^{\mathbb{Z} / 4}\left(P_{2}(x)\right)\right]=\left[\beta^{\mathbb{Z} / 2}\left(x^{2}\right)\right]=0,
$$

since $N$ is $\operatorname{spin}^{c}$. However $\widetilde{Q}(x) \neq 0$, since any element of $\alpha H^{2}(N)$ is 2-torsion, while

$$
2 \beta^{\mathbb{Z} / 4}\left(P_{2}(x)\right)=\beta^{\mathbb{Z} / 2}\left(x^{2}\right) \neq 0 .
$$

Hence $\operatorname{ord}(\widetilde{Q}(x))=2$ and we're done.

6-manifolds violating the TPIC. In this subsection we give examples of Teichner pairs $(N, x)$ over a nonorientable base which violate the Topological Period-Index Conjecture, i.e., $\beta^{\mathbb{Z} / 2}\left(x^{2}\right) \notin \beta^{\mathbb{Z} / 2}(x) H^{2}(N)$. We first prove an extension of [Teichner 1995, Lemma 2]. 
Lemma 5.6. Let $M$ be a closed connected 4-manifold with an element $a \in H_{1}(M)$ of order 4. Then there exists a 3-dimensional bundle $E$ over $M$ with $w_{1}(E)=$ $w_{1}(M), w_{2}(E)$ not coming from $H^{2}\left(M ; \mathbb{Z}^{w_{1}(E)}\right)$, and $w_{3}(E)=0$.

Proof. We use multiplicative notation for elements of $H_{1}(M)=\pi_{1}(M)_{\mathrm{ab}}$. The Poincaré dual of $a^{2}$ in $H^{3}\left(M ; \mathbb{Z}^{w_{1}(M)}\right)$ has order 2 and hence is the image of an element $z \in H^{2}(M ; \mathbb{Z} / 2)$ under the twisted Bockstein. As in Teichner's proof of [1995, Lemma 2], there are no obstructions to constructing a 3-bundle $E$ with $\left(w_{1}(E), w_{2}(E)\right)=\left(w_{1}(M), z\right)$.

It remains to show that $w_{3}(E)=0$. This follows from Theorem 2.3 of [Greenblatt 2006], which states that for any space $X$ and twisting $w \in H^{1}(X ; \mathbb{Z} / 2)$, the composition of the twisted Bockstein $\beta^{w}: H^{i}(X ; \mathbb{Z} / 2) \rightarrow H^{i+1}\left(X ; \mathbb{Z}^{w}\right)$ with reduction mod 2 is given by

$$
\rho_{2} \circ \beta^{w}(z)=S q^{1}(z)+z w .
$$

Hence we have

$$
\begin{aligned}
w_{3}(E) & =S q^{1}\left(w_{2}(E)\right)+w_{2}(E) w_{1}(E) \\
& =\rho_{2} \circ \beta^{w_{1}(M)}\left(w_{2}(E)\right) \\
& =0
\end{aligned}
$$

since $\beta^{w_{1}(M)}\left(w_{2}(E)\right)=\beta^{w_{1}(M)}(z)$ is even.

In order to find an example with $\beta^{\mathbb{Z} / 2}\left(x^{2}\right) \notin \beta^{\mathbb{Z} / 2}(x) H^{2}(N)$ it turns out to be sufficient that there is an element $a \in H_{1}(M)$ of order 4 such that $0 \neq \tau_{!}\left(a^{2}\right) \in$ $H_{1}(\widehat{M})$, where $\tau_{!}: H_{1}(M) \rightarrow H_{1}(\widehat{M})$ is the transfer associated to the orientation double cover $\tau: \widehat{M} \rightarrow M$.

To this end, we shall use a closed connected 4-manifold $M$ with

$$
\pi_{1}(M)=C_{8} \rtimes C_{2}=\left\langle a, b \mid b^{-1} a b=a^{5}, a^{8}, b^{2}\right\rangle
$$

and with $w_{1}(M): \pi_{1}(M) \rightarrow C_{2}$ the projection onto the base of the semidirect product. Note that

$$
H_{1}(M)=\left\langle a, b \mid a=a^{5}, a^{8}, b^{2},[a, b]\right\rangle \cong C_{4} \times C_{2}
$$

has an element $a$ of order 4. It is well known (see, e.g., [Ranicki 2002, Propostion $11.75])$ that every homomorphism $w: \pi \rightarrow \mathbb{Z} / 2$ from a finitely presented group $\pi$ arises as $\left(\pi_{1}(X), w_{1}(X)\right)$ for a 4-manifold $X$, so a 4-manifold $M$ as above exists.

Lemma 5.7. The transfer homomorphism $\tau_{!}: H_{1}(M) \rightarrow H_{1}(\widehat{M})$ does not map the element $a^{2} \in H_{1}(M)$ to 0 .

Proof. Let $G=\pi_{1}(M)$ and let $H=\operatorname{ker}\left(w_{1}(M)\right)=C_{8}$, so that $[G: H]=2$. The definition of the transfer in terms of coset representatives gives

$$
\tau_{!}: G_{\mathrm{ab}} \rightarrow H_{\mathrm{ab}}, \quad g[G, G] \mapsto g^{2}[H, H] .
$$

Therefore $\tau_{!}\left(a^{2}\right)=a^{4} \neq 0$ as claimed. 
Before continuing, we record the following lemma which will be useful in the proof of Proposition 5.9 below.

Lemma 5.8 [Dold 1980, Chapter VII, §8.10]. Let $i: A \rightarrow X$ denote the inclusion of a $C W$-pair $(X, A)$, and let $\delta: H^{*}(A) \rightarrow H^{*+1}(X, A)$ be the connecting homomorphism in the long exact cohomology sequence (with any coefficients). Then for all $x \in H^{*}(A)$ and $y \in H^{*}(X)$ we have

$$
\delta\left(x i^{*}(y)\right)=\delta(x) y .
$$

The following proposition proves Theorem 1.4(ii).

Proposition 5.9. Let $(N, x)$ be a Teichner pair over a nonorientable 4-manifold $M$ with $w_{1}(M): \pi_{1}(M) \rightarrow \mathbb{Z} / 2$ as above. Then $\beta^{\mathbb{Z} / 2}\left(x^{2}\right) \notin \beta^{\mathbb{Z} / 2}(x) H^{2}(N)$.

Furthermore, the element $\alpha=\beta^{\mathbb{Z} / 2}(x) \in T H^{3}(N)$ has $\operatorname{per}(\alpha)=2$ and $\operatorname{ind}(\alpha)=8$.

Proof. We first prove that $\beta^{\mathbb{Z} / 2}\left(x^{2}\right) \notin \beta^{\mathbb{Z} / 2}(x) H^{2}(N)$. Suppose towards a contradiction that $\beta^{\mathbb{Z} / 2}\left(x^{2}\right)=\beta^{\mathbb{Z} / 2}(x) Y$ for some $Y \in H^{2}(N)$. Let $i: N \hookrightarrow D E$ be the inclusion of the unit sphere bundle in the unit disc bundle of $E$. From the long exact sequence of the pair $(D E, N)$, the twisted Thom isomorphism $H^{3}(D E, N) \cong$ $H^{0}\left(M ; \mathbb{Z}^{w}\right)$, and the fact that $M$ is nonorientable, we see that $i^{*}: H^{2}(D E) \rightarrow$ $H^{2}(N)$ is surjective. Hence $Y=i^{*}(y)$ for some $y \in H^{2}(D E) \cong H^{2}(M)$.

Let $t_{E}^{w} \in H^{3}\left(D E, N ; \mathbb{Z}^{w}\right)$ be the twisted Thom class of $E$ and $t_{E} \in H^{3}(D E, N ; \mathbb{Z} / 2)$ its mod 2 reduction. From the fact that $x$ restricts to a generator in each fibre, it follows that $t_{E}=\delta(x)$, where $\delta: H^{*}(N ; \mathbb{Z} / 2) \rightarrow H^{*+1}(D E, N ; \mathbb{Z} / 2)$ is the connecting homomorphism (see the proof of Lemma 1 in [Teichner 1995]). Now we have

$$
\delta\left(x^{2}\right)=\delta\left(S q^{2}(x)\right)=S q^{2}(\delta(x))=S q^{2}\left(t_{E}\right)=w_{2}(E) t_{E}
$$

and since Bocksteins commute with connecting homomorphisms

$$
\delta\left(\beta^{\mathbb{Z} / 2}\left(x^{2}\right)\right)=\beta^{\mathbb{Z} / 2}\left(\delta\left(x^{2}\right)\right)=\beta^{\mathbb{Z} / 2}\left(w_{2}(E) t_{E}\right) .
$$

On the other hand, $\beta^{\mathbb{Z} / 2}\left(x^{2}\right)=\beta^{\mathbb{Z} / 2}(x) i^{*}(y)$ and so

$$
\begin{aligned}
\delta\left(\beta^{\mathbb{Z} / 2}\left(x^{2}\right)\right) & =\delta\left(\beta^{\mathbb{Z} / 2}(x) i^{*}(y)\right) \\
& =\delta\left(\beta^{\mathbb{Z} / 2}\left(x i^{*}\left(\rho_{2}(y)\right)\right)\right) \\
& =\beta^{\mathbb{Z} / 2}\left(\delta\left(x i^{*}\left(\rho_{2}(y)\right)\right)\right) \\
& =\beta^{\mathbb{Z} / 2}\left(\delta(x) \rho_{2}(y)\right) \\
& =\beta^{\mathbb{Z} / 2}\left(t_{E} \rho_{2}(y)\right) .
\end{aligned}
$$

Here we have used Lemmas 3.1 and 5.8. 
The above argument shows that $\beta^{\mathbb{Z} / 2}\left(w_{2}(E) t_{E}\right)=\beta^{\mathbb{Z} / 2}\left(t_{E} \rho_{2}(y)\right)$, or equivalently $t_{E}\left(w_{2}(E)-\rho_{2}(y)\right)$ is the reduction of an integral class. From the square

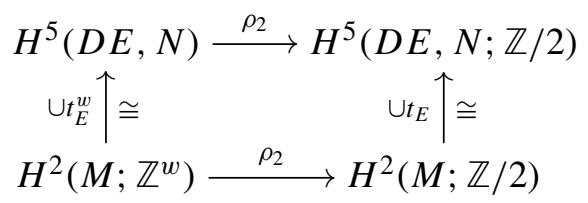

which commutes since the Thom isomorphisms commute with reduction mod 2, we see that $w_{2}(E)-\rho_{2}(y)$ is the reduction of a twisted integral class, or equivalently

$$
\beta^{w}\left(w_{2}(E)\right)=\beta^{w}\left(\rho_{2}(y)\right) .
$$

Next we lift this equation to the orientation cover, using the commutative square

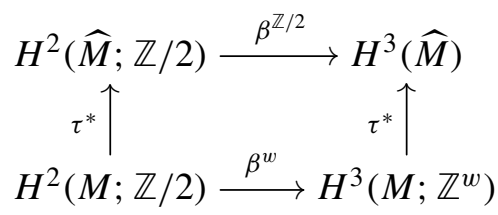

to conclude that

$$
\tau^{*} \beta^{w}\left(w_{2}(E)\right)=\tau^{*} \beta^{w}\left(\rho_{2}(y)\right)=\beta^{\mathbb{Z} / 2}\left(\tau^{*}\left(\rho_{2}(y)\right)\right)=\beta^{\mathbb{Z} / 2} \rho_{2}\left(\tau^{*}(y)\right)=0 .
$$

However, Poincaré duality gives a commutative square

$$
\begin{array}{crl}
H^{3}\left(M ; \mathbb{Z}^{w}\right) \stackrel{\tau^{*}}{\longrightarrow} & H^{3}(\widehat{M}) \\
\cap[M]_{w} \underset{\downarrow}{ } & \cap[\widehat{M}]\rfloor \cong \\
H_{1}(M) \stackrel{\tau_{!}}{\longrightarrow} H_{1}(\widehat{M})
\end{array}
$$

Since the bundle $E$ was chosen as in Lemma 5.6 so that $\beta^{w}\left(w_{2}(E)\right) \cap[M]_{w}=a^{2}$, and $\tau_{!}\left(a^{2}\right) \neq 0$ by Lemma 5.7, we see that $\tau^{*} \beta^{w}\left(w_{2}(E)\right) \neq 0$, a contradiction.

To prove the second statement, we have $\operatorname{per}(\alpha)=2$ and since $\beta^{\mathbb{Z} / 2}\left(x^{2}\right) \notin$ $\beta^{\mathbb{Z} / 2}(x) H^{2}(N)$,

$$
2 \widetilde{Q}(x)=\left[\beta^{\mathbb{Z} / 4}\left(P_{2}(x)\right)\right]=\left[2 \beta^{\mathbb{Z} / 4}\left(P_{2}(x)\right)\right]=\left[\beta^{\mathbb{Z} / 2}\left(x^{2}\right)\right] \neq 0 .
$$

Hence $\operatorname{ord}(\widetilde{Q}(x))=4$. As $\operatorname{ind}(\alpha)=\operatorname{ord}(\widetilde{Q}(x)) \operatorname{per}(\alpha)$ by [Antieau and Williams 2014 b, Theorem A], ind $(\alpha)=8$.

\section{Acknowledgements}

We would like to thank Ben Antieau and Ben Williams for helpful comments and for pointing out mistakes in early stages of our work, Ben Martin for providing a proof of Lemma 3.5, Christian Haesemeyer for guidance with the Algebraic PeriodIndex Conjecture, and Jim Davis and Mark Powell for advice regarding the linking pairing. Finally we thank the anonymous referee of an earlier version of this paper for comments which have improved the exposition. 


\section{References}

[Antieau and Williams 2014a] B. Antieau and B. Williams, "The period-index problem for twisted topological K-theory”, Geom. Topol. 18:2 (2014), 1115-1148. MR Zbl

[Antieau and Williams 2014b] B. Antieau and B. Williams, "The topological period-index problem over 6-complexes", J. Topol. 7:3 (2014), 617-640. MR Zbl

[Brown 1982] K. S. Brown, Cohomology of groups, Graduate Texts in Mathematics 87, Springer, 1982. MR Zbl

[Colliot-Thélène 2002] J.-L. Colliot-Thélène, "Exposant et indice d'algèbres simples centrales non ramifiées", Enseign. Math. (2) 48:1-2 (2002), 127-146. MR Zbl

[Davis and Kirk 2001] J. F. Davis and P. Kirk, Lecture notes in algebraic topology, Graduate Studies in Mathematics 35, American Mathematical Society, Providence, RI, 2001. MR Zbl

[Dold 1980] A. Dold, Lectures on algebraic topology, 2nd ed., Grundlehren der Mathematischen Wissenschaften 200, Springer, 1980. MR Zbl

[Donovan and Karoubi 1970] P. Donovan and M. Karoubi, "Graded Brauer groups and $K$-theory with local coefficients”, Inst. Hautes Études Sci. Publ. Math. 38 (1970), 5-25. MR Zbl

[Grant and Szúcs 2013] M. Grant and A. Szúcs, "On realizing homology classes by maps of restricted complexity”, Bull. Lond. Math. Soc. 45:2 (2013), 329-340. MR Zbl

[Greenblatt 2006] R. Greenblatt, "Homology with local coefficients and characteristic classes", Homology Homotopy Appl. 8:2 (2006), 91-103. MR Zbl

[Grothendieck 1968] A. Grothendieck, "Le groupe de Brauer, I: Algèbres d'Azumaya et interprétations diverses", pp. 46-66 in Dix exposés sur la cohomologie des schémas, Adv. Stud. Pure Math. 3, North-Holland, Amsterdam, 1968. MR Zbl

[Gu 2019] X. Gu, "The topological period-index problem over 8-complexes, I", J. Topol. 12:4 (2019), 1368-1395. Zbl

[de Jong 2004] A. J. de Jong, "The period-index problem for the Brauer group of an algebraic surface", Duke Math. J. 123:1 (2004), 71-94. MR Zbl

[Massey 1961] W. S. Massey, "Obstructions to the existence of almost complex structures", Bull. Amer. Math. Soc. 67 (1961), 559-564. MR Zbl

[Morgan 1996] J. W. Morgan, The Seiberg-Witten equations and applications to the topology of smooth four-manifolds, Mathematical Notes 44, Princeton University, 1996. MR Zbl

[Ranicki 2002] A. Ranicki, Algebraic and geometric surgery, Oxford University, 2002. MR Zbl

[Seifert and Threlfall 1934] H. Seifert and W. Threlfall, Lehrbuch der Topologie, Teubner, Leipzig, 1934. Zbl

[Teichner 1995] P. Teichner, "6-dimensional manifolds without totally algebraic homology", Proc. Amer. Math. Soc. 123:9 (1995), 2909-2914. MR Zbl

[Thom 1954] R. Thom, "Quelques propriétés globales des variétés différentiables", Comment. Math. Helv. 28 (1954), 17-86. MR Zbl

Received 4 Feb 2020. Revised 10 Feb 2020. Accepted 25 Feb 2020.

DIARMUID CROWLEY: dcrowley@unimelb.edu.au

School of Mathematics and Statistics, The University of Melbourne, Parkville, Australia

MARK GRANT: mark.grant@abdn.ac.uk

Institute of Mathematics, University of Aberdeen, Aberdeen, United Kingdom 


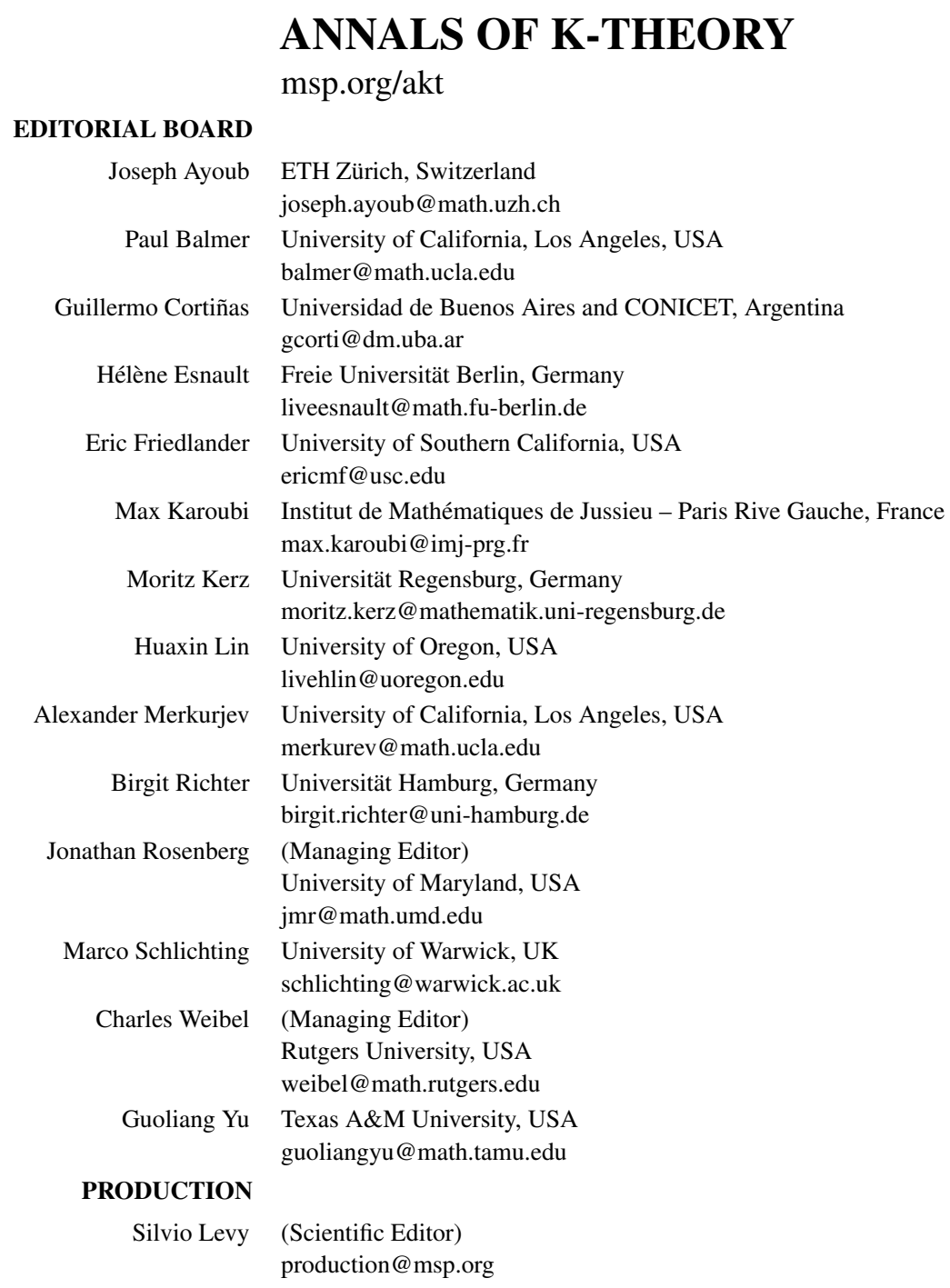

Annals of K-Theory is a journal of the K-Theory Foundation (ktheoryfoundation.org). The K-Theory Foundation acknowledges the precious support of Foundation Compositio Mathematica, whose help has been instrumental in the launch of the Annals of K-Theory.

See inside back cover or msp.org/akt for submission instructions.

The subscription price for 2020 is US \$510/year for the electronic version, and $\$ 575 /$ year $(+\$ 25$, if shipping outside the US) for print and electronic. Subscriptions, requests for back issues and changes of subscriber address should be sent to MSP.

Annals of K-Theory (ISSN 2379-1681 electronic, 2379-1683 printed) at Mathematical Sciences Publishers, 798 Evans Hall \#3840, c/o University of California, Berkeley, CA 94720-3840 is published continuously online. Periodical rate postage paid at Berkeley, CA 94704, and additional mailing offices.

AKT peer review and production are managed by EditFlow ${ }^{\circledR}$ from MSP.

\section{PUBLISHED BY}

mathematical sciences publishers

nonprofit scientific publishing

http://msp.org/

(C) 2020 Mathematical Sciences Publishers 


\section{ANNALS}

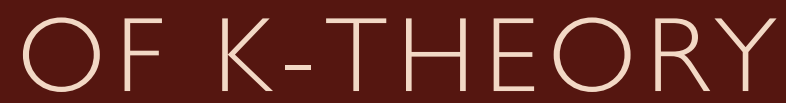

2020

vol. 5

no. 3

Coassembly is a homotopy limit map

CARy Malkiewich and Mona Merling

Rational equivalence of cusps

395

SHOUHEI MA

$C_{2}$-equivariant stable homotopy from real motivic stable homotopy

MARK BEHRENS and JAY SHAH

Groups with Spanier-Whitehead duality

465

Shintaro NishikaWA and VALERIo Proietti

Homotopy equivalence in unbounded $K K$-theory

501

Koen VAn Den Dungen and BRAm Mesland

The $p$-completed cyclotomic trace in degree 2

JoHANNES ANSCHÜTZ and ARTHUR-CÉSAR LE BRAS

Nisnevich topology with modulus

HIROYASU MIYAZAKI

The Topological Period-Index Conjecture for $\operatorname{spin}^{c}$ 6-manifolds

605

DIARMUID CROWLEY and MARK GRANT

Weibel's conjecture for twisted $K$-theory

JoEl STAPLETON

Positive scalar curvature metrics via end-periodic manifolds

639

Michael Hallam and VARghese Mathai 


\section{University Library}

\section{- M M I N E R VA A gateway to Melbourne's research publications}

Minerva Access is the Institutional Repository of The University of Melbourne

Author/s:

Crowley, D;Grant, M

Title:

The Topological Period-Index Conjecture for spin(c) 6-manifolds

Date:

2020-01-01

Citation:

Crowley, D. \& Grant, M. (2020). The Topological Period-Index Conjecture for spin(c) 6-manifolds. ANNALS OF K-THEORY, 5 (3), pp.605-620. https://doi.org/10.2140/ akt.2020.5.605.

Persistent Link:

http://hdl.handle.net/11343/254324 